\title{
GEOMETRICAL PROPERTIES OF CONES AND THE COMPARISON OF SOLUTIONS OF DIFFERENTIAL EQUATIONS
}

\author{
YULY A. SAET
}

\begin{abstract}
We introduce here certain geometrical concepts related to the properties of cones in Banach spaces and show that these definitions are realizable. Then the results on the comparison of solutions are formulated where the ordering is introduced by means of a cone which is not required to be solid.
\end{abstract}

Introduction. The concept of quasimonotonicity, introduced in [1], has been extended and generalized in different ways. Whenever a partial ordering was introduced by means of a cone, either the solidity of the cone or strong restrictions on the relevant differential equations were required. The problem of the existence of differential equations in arbitrary closed sets is treated in [7]. However, when the set is a cone, the theorems of comparison (or invariance) found in [ 3 and 7], as well as in [8], involve either Lipschitz-like conditions, or the presence of interior points, even if questions of existence are treated separately from those of invariance. The distinction of the present approach from those publications known to the author lies in the domain of "boundary conditions" [7] which in [2,4 and 8] are imposed on the whole cone if the cone is not solid. Here we investigate the possibility of comparing solutions for nonsolid cones and our "boundary conditions" are imposed only on a subset of the cone. Our goal is to introduce and illustrate the use of a certain geometrical concept. For that reason we select the simplest type of additional "boundary conditions" on the differential equation itself. They also have intrinsic geometrical significance in our situation, but for the solid cone case analogous conditions were used earlier in [3]. All terms used hereafter without any explanations have the same meanings as in [6].

1. Let $B$ be a real Banach space and $P$ a cone in $B$. An element $u \in P$ is called a relatively-interior one if a neighborhood $R_{u}$ of the zero element in $B$ exists such that for arbitrary $x \in R_{u} \cap P$ the element $(u-x) \in P$. A cone is called presolid if it contains a relatively-interior point. Let $P$ be a presolid cone; then the set $W$ of nonrelatively interior elements of $P$ is called the wall of the cone.

REMARK. The above definition of relatively-interior element is equivalent to the following: The element $x \in P$ is relatively-interior iff $\forall \phi \in P, \exists \mu>0$ such that $(x-\mu \phi) \in P$.

Received by the editors August 7, 1980 and, in revised form, November 16, 1981, September 20, 1982. 1980 Mathematics Subject Classification. Primary 46A40, 46B20, 34K30.

Key words and phrases. Cone in Banach space, positive solution, differential inequality. 
Proof. Given $\phi \in P, \exists n \in N$ such that $\left(x-n^{-1} \phi\right) \in P$, i.e., $\phi \in n(x-P)$. Thus

$$
P=\bigcup_{n=1}^{\infty}[n(x-P) \cap P] .
$$

Notice that $P$ and $n(x-P)$ are closed sets in the complete metric space $B$. Thus, by Baire's theorem, $\exists \phi_{0} \in P$ and a real number $s>0$ such that

$$
\phi_{0}+P_{s} \subset x-P
$$

where $P_{s}=\{h \in P:\|h\|<s\}$. Rewrite (1) as $x-\phi_{0}-P_{s} \subset P$. Then we have

$$
x-P_{s}=\left(x-\phi_{0}-P_{s}\right)+\phi_{0} \subset P \text {, }
$$

as was required.

Let us show that there are Banach spaces with presolid cones having some other useful properties.

THEOREM. There exists a real Banach space $E$ with a cone $K$ which satisfies the following conditions:

(a) the linear hull of $K$ is dense in the space $E$;

(b) the cone $K$ is normal;

(c) the cone $K$ is not solid;

(d) the cone $K$ is minihedral;

(e) an element $u \in K$ exists such that $x \in K,\|x\| \leqslant 1 \Rightarrow(u-x) \in K$.

Thus the cone $K$ is presolid.

Proof. Let $E$ be the set of all sequences of real numbers $x=\left(x_{1}, x_{2}, \ldots, x_{n}, \ldots\right)$, such that

$$
\|x\| \equiv \sup \left\{\left|x_{1}\right|,\left|x_{1}+x_{2}\right|,\left|x_{2}+x_{3}\right|, \ldots,\left|x_{n-1}+x_{n}\right|, \ldots\right\}
$$

is finite. With coordinatewise operations the set $E$ is a real linear space and it is clear that $\|\cdot\|$ possesses the properties of a norm. It follows directly from the definition of the norm that convergence in this norm means coordinatewise convergence. Routine maneuvers then confirm that the space $E$ is complete in this norm. Thus, $E$ is a Banach space. Let $K$ be the set of all elements $x \in E, x=\left\{x_{k}\right\}(k=1,2, \ldots)$ such that for all $k, x_{k} \geqslant 0$ and $x_{k} \leqslant N_{x}$, where $N_{x}$ is a positive number depending only on the element $x$. Let $m$ be the normed space of bounded number sequences. The set $K$ is a subset of the set $m, K \subset m$. On the other hand the set $m$ is a part of the set $E, m \subset E$. Let us denote by $\|\cdot\|_{m}$ the norm in the space $m$. Then for all $x \in K$,

$$
\|x\|_{m} \leqslant\|x\| \leqslant 2\|x\|_{m} .
$$

The set $K$ is closed in the space $m$ and therefore it is closed in the space $E$ also. It follows from this fact that $K$ is a cone in the space $E$. Let us denote by $X$ the closure of the set $m$ in the norm $\|\cdot\|$ of the space $E . X$ is a subspace of the space $E$. Thus, now the set $K$ becomes a cone in the Banach space $X$. Let $x, y \in K, x=\left\{x_{i}\right\}$, $y=\left\{y_{i}\right\}$. Let us form the sequence $z=\left\{z_{i}\right\}, z_{i}=\max \left(x_{i}, y_{i}\right)$. Then $z \in X$ and $z=\sup (x, y)$. Therefore, part (d) of the theorem is proved. Let $x_{1}, x_{2} \in K$, $\left\|x_{1}\right\|=\left\|x_{2}\right\|=1$. Then from (2) it follows that $\left\|x_{1}+x_{2}\right\| \geqslant\left\|x_{1}+x_{2}\right\|_{m}>1$, and thus part (b) is proved. 
Let us take the element $u=(1,1, \ldots, 1, \ldots) \in K$ and consider any element $x \in K$ with $\|x\| \leqslant 1$. Then it follows from (2) that $(u-x) \geqslant 0$. Thus, part (e) is proved. We will prove (c) by reductio ad absurdum. To this end let us suppose that $K$ is a solid cone in $E$. Then $K$ is a reproducing cone and the set $X$ must coincide with the set $m$. From the definition of the norms $\|\cdot\|_{m}$ and $\|\cdot\|$ it then follows that $\|x\| \leqslant 2\|x\|_{m} \forall x \in X$.

Now we appeal to the following consequence of Banach's theorem on the inverse operator: If in a linear space two norms are given which convert this space into Banach spaces $E_{1}$ and $E_{2}$, and in addition there is a positive number $C_{1}$ such that $\|x\|_{2} \leqslant C_{1}\|x\|_{1}$ for all $x \in E$, then the norms $\|\cdot\|_{1}$ and $\|\cdot\|_{2}$ are equivalent. Accordingly, the norms $\|\cdot\|$ and $\|\cdot\|_{m}$ must be equivalent in the space $m$. Let us show that this is absurd. Let $n$ be an arbitrary natural number and define the elements

$$
\begin{array}{r}
x_{n}=(1,-2,3,-4,5, \ldots,-2 n, 2 n-1,2 n-2,2 n-3, \ldots, 1,0,0, \ldots), \\
n=1,2, \ldots .
\end{array}
$$

Then $x_{n} \in m,\left\|x_{n}\right\|_{m}=2 n$ and $\left\|x_{n}\right\|=1$, for each $n$. This is a contradiction. Thus, part (c) of the theorem is proved.

Inasmuch as the convergence in $E$ is coordinatewise, the linear hull of $K$ is dense in $E$. That proves part (a), so the theorem is completely proved.

2. Let $f(x, t)$ be a continuous function defined on $B \times J$ with values in $B$, where $B$ is a real Banach space and $J$ is the interval $0 \leqslant t \leqslant T$ with $0<T \leqslant \infty$. By the solution of the initial-value problem

$$
d x / d t=f(x, t), \quad x(0)=x_{0},
$$

on $[0, a), a \leqslant T$, we mean the function $x(t)$ having a strong derivative on that interval and satisfying the differential equation and the initial condition.

Let $K$ be a presolid cone in space $B$ and $L(K)$ the linear hull of $K$. Suppose the functions $f_{1}(x, t)$ and $f_{2}(x, t)$ have the properties mentioned above for $f(x, t)$, and the following conditions are satisfied:

$$
F(p, x, t) \in L(K) \quad \forall p \in K, x \in B, t \in[0, a),
$$

where

$$
F(p, x, t) \equiv f_{1}(x+p, t)-f_{2}(x, t)
$$

and

$$
F(p, x, t)+M p \in K_{\text {rip }} \quad \forall p \in W, x \in B, t \in[0, a) .
$$

Here $K_{\text {rip }}$ is the set of relatively interior points of $K, W$ is a wall of $K$ and $M$ is a positive real number.

Let us consider now two initial-value problems

$$
d x_{i} / d t=f_{i}\left(x_{i}, t\right), \quad x_{i}(0)=x_{i 0} \quad(i=1,2) .
$$

Suppose that $x_{i}(t)$ are solutions of these problems on the interval $[0, a)$.

THEOREM. Let conditions (3) and (4) be valid and let $x_{10} \geqslant x_{20}$. Then $x_{1}(t) \geqslant x_{2}(t)$ for $t \in[0, a)$. 
Proof. Let us denote by $p(t)$ the function

$$
p(t) \equiv x_{1}(t)-x_{2}(t), \quad t \in[0, a),
$$

and define $p_{0}=x_{10}-x_{20}$.

The function $p(t)$ satisfies the differential equation $d p / d t=F(p, t)$, where $F(p, t) \equiv f_{1}\left[x_{2}(t)+p, t\right]-f_{2}\left[x_{2}(t), t\right]$, and the initial condition $p(0)=p_{0} \in K$.

Let us denote by $t$ the supremum of the set

$$
\Omega=\{s: p(t) \geqslant 0 \text { for all } t \in[0, s], 0 \leqslant s<a\} .
$$

What must be proved is that $\bar{t}=a$.

Assume that $\bar{t}<a$. Then all the values $p(t)$ belong to the set $K$ on the entire interval $[0, \bar{t}]$. Now we denote $p_{\mathrm{cr}}=p(\bar{t})$ and use the formula

$$
p(\bar{t}+\tau)=p_{\mathrm{cr}}+\tau \cdot F\left(p_{\mathrm{cr}}, \bar{t}\right)+\tau \cdot \beta(\tau)
$$

from [5], where $\|\beta(\tau)\| \rightarrow 0$ as $\tau \rightarrow 0$ and $\beta(\tau) \in L(K)$ for small $\tau>0$ by virtue of condition (3). The element $p_{\text {cr }}$ cannot belong to $K_{\text {rip }}$, since otherwise for sufficiently small positive $\tau$ for all $t \in[\bar{t}, \bar{t}+\tau]$ we would have $p(t) \in K$, contrary to the definition of the number $\bar{t}$. Thus $p_{\mathrm{cr}} \in W$. Now we make use of hypothesis (4) of the theorem. We have

$$
p(\bar{t}+\tau)=\left(p_{\mathrm{cr}}+\tau \cdot F\left(p_{\mathrm{cr}}, t\right)+\tau M p_{\mathrm{cr}}\right)-\tau M p_{\mathrm{cr}}+\tau \cdot \beta(\tau) .
$$

Here the element $\left(p_{\mathrm{cr}}+\tau \cdot F\left(p_{\mathrm{cr}}, \bar{t}\right)+\tau M p_{\mathrm{cr}}\right)$ is a relatively interior element of $K$.

Consequently, for small positive $\tau$ the elements $p(\bar{t}+\tau)$ belong to $K$. This violates the definition of $t$ and therefore the theorem is proved.

Acknowledgement. The author is indebted to Professor S. Saeki of Tokyo Metropolitan University for the proof of the fact in the remark.

\section{REFERENCES}

1. M. Müller, Über das Fundamental Theorem in der Theorie gewöhnlichen Differentialgleichungen, Math. Z. 26 (1927), 619-645.

2. J. Sharskii, Differential inequalities, PAN, Warsaw, 1971.

3. W. Walter, Ordinary differential inequalities in ordered Banach spaces, J. Differential Equations 9 (1971), 253-261. Differential and integral inequalities, Springer-Verlag, Berlin, Heidelberg and New York. 1970.

5. L. Schwartz, Analyse mathématique, Hermann, Paris, 1967.

6. M. Krasnoselskii, Positive solutions of operator equations, Noordhoff, Groningen, 1964.

7. K. Deimling, Ordinary differential equations in Banach spaces, Springer-Verlag, Berlin, Heidelberg and New York, 1977.

8. R. H. Martin, Jr., Nonlinear operators and differential equations in Banach spaces, Wiley, New York, 1976.

Department of Mathematics, Lamar University, Beaumont, TeXas 77710 